\title{
On the desirability of models for inferring genome phylogenies
}

\section{James O. Mclnerney}

Department of Biology, National University of Ireland, Maynooth, County Kildare, Ireland

Genomes are clearly suited for inferring common ancestry and for understanding ancestor-descendent relationships and interspecies gene transfer. Genomic evolutionary models can tell us a great deal about the processes that drive genome evolution, the mutational and selective pressures that lead to the genesis of biochemical pathways and operons, and the nature and extent of lateral gene transfer (LGT). Simultaneously, a robust phylogeny can be constructed that depicts the evolutionary relationships of the organisms in which the genomes are found.

Several approaches have been employed to infer species phylogenies at the genome level. In general terms, these can be divided into ad hoc summary statistics based on genome content, the use of concatenated alignments and the use of consensus methods (i.e. phylogenetic supertrees [1]).

The basic premise of methods based on summary statistics is that genomes are compared and a gene content matrix is compiled. Then, either a distance is estimated between all pairs of taxa and entered into a distance matrix that is summarized using a clustering algorithm, or a dendrogram is inferred using maximum parsimony. This is usually referred to as the species phylogeny. The principal difference between this approach and the approaches that use concatenated alignments or supertrees is that information concerning homolog interrelationships is not used. Presence or absence of homologs is the only information that is scored, and this approach can be considered ad hoc in the sense that the methods are applied uniformly to all datasets and, therefore, the assumptions are not informed by the data themselves.

Unsurprisingly, the results of using summary statistics have been variable. Although many methods have recovered groups that seem sensible and have support from external biochemical or morphological data, there have been cases in which the inferred trees are unusual [2].

For example, the haloarchaea are a group of halophilic Archaea, long taken to be members of the Euryarchaeota. Wolf et al. [2] and Korbel et al. [3] placed this taxon at the base of the Archaea. In the figures of Henz et al. [4], this taxon was placed among the Bacteria in one instance, within the Euryarchaeota in a second example and as the deepest-branching Archaeon in a third example. Dutilh et al. [5] point out that the correct

Corresponding author: McInerney, J.O. (james.o.mcinerney@nuim.ie).

Available online 3 November 2005

www.sciencedirect.com placement of the haloarchaea is within the Euryarchaeota and that previous methods placed this taxon erroneously as a deep-branching Archaeon. This erroneous placement is likely to be due to the large number of bacterial genes present in the haloarchaea [6]. The haloarchaea are, therefore, pulled to a position that is intermediate between the two groups from which the haloarchaea genes came. The data violate the ad hoc assumptions of the methods. Problems of this nature argue for the development of explicit genome evolutionary models.

Evolutionary models are statements concerning how it is thought that evolution has occurred [7]. If a model were correct, the inferred distances between two genomes would be accurate and would provide consistent estimates of the topology of the resulting phylogeny. The most desirable properties of these models are explicitness when describing the evolutionary process, realism or plausibility of the assumptions contained in the models and clarity in the interpretation of the output [8]. Usually, models are derived in a maximum likelihood framework in which the model consists of the phylogenetic tree of the genomes and the process underlying their evolution [9]. However, even when alternative models are not tested or lengthy computational optimization is not performed, an explicit model of evolution can still be assumed in calculations [10].

A realistic model of genome evolution must, as a minimum, deal with gene duplication and loss, in addition to acquisition of genes by LGT. This is not to say that all parameters are necessary for all analyses. When models differ in their numbers of free parameters and are nested, a likelihood ratio test can be used to choose the most appropriate parameter.

Gu and Zhang [11] describe a model called the extended genome content distance. This model uses the number of homologs $(0,1$ or $>1)$ to derive the genome distance. The model does not take account of horizontal gene transfer and, as a result, the authors report a position for the haloarchaea that is the same as the much simpler method of Korbel et al. [3]. A model has also been developed that deals with LGT, albeit in a slightly different setting [12]. Nonetheless, the development of explicit model-based approaches is to be welcomed as a useful step towards the understanding of genome evolution.

When the genomic age began, it was assumed that the huge increase in the amount of available data would result in more-accurate phylogenies. Instead, the extent of apparent genome plasticity has fueled a passionate debate 
concerning prokaryotic evolution. Sensible genome models that provide information about phylogeny and the process of evolution should be a goal for genomics and systematics.

\section{References}

1 Creevey, C.J. and McInerney, J.O. (2005) Clann: investigating phylogenetic information through supertree analyses. Bioinformatics 21, 390-392

2 Wolf, Y.I. et al. (2001) Genome trees constructed using five different approaches suggest new major bacterial clades. BMC Evol. Biol. 1, 8

3 Korbel, J.O. et al. (2002) SHOT: a web server for the construction of genome phylogenies. Trends Genet. 18, 158-162

$4 \mathrm{Henz}$, S.R. et al. (2005) Whole-genome prokaryotic phylogeny. Bioinformatics 21, 2329-2335

5 Dutilh, B.E. et al. (2004) The consistent phylogenetic signal in genome trees revealed by reducing the impact of noise. J. Mol. Evol. $58,527-539$

6 Kennedy, S.P. et al. (2001) Understanding the adaptation of Halobacterium species NRC-1 to its extreme environment through computational analysis of its genome sequence. Genome Res. 11, 1641-1650

7 Felsenstein, J. (1988) Phylogenies from molecular sequences: inference and reliability. Annu. Rev. Genet. 22, 521-565

8 Huelsenbeck, J.P. and Crandall, K.A. (1997) Phylogeny estimation and hypothesis testing using maximum likelihood. Annu. Rev. Ecol. Syst. 28, 437-466

9 Edwards, A.W.F. (1972) Likelihood, 2nd edn, Johns Hopkins University Press

10 Lake, J.A. and Rivera, M.C. (2004) Deriving the genomic tree of life in the presence of horizontal gene transfer: conditioned reconstruction. Mol. Biol. Evol. 21, 681-690

$11 \mathrm{Gu}, \mathrm{X}$. and Zhang, H. (2004) Genome phylogenetic analysis based on extended gene contents. Mol. Biol. Evol. 21, 1401-1408

12 Novozhilov, A.S. et al. (2005) Mathematical modeling of evolution of horizontally transferred genes. Mol. Biol. Evol. $22,1721-1732$

\title{
Bifunctional TatA subunits in minimal Tat protein translocases
}

\section{Jan D.H. Jongbloed ${ }^{1,2}$, René van der Ploeg ${ }^{3}$ and Jan Maarten van Dijl ${ }^{3}$}

\author{
${ }^{1}$ Department of Genetics, Groningen Biomolecular Sciences and Biotechnology Institute, Kerklaan 30, 9751 NN Haren, \\ The Netherlands \\ ${ }^{2}$ Present address: Department of Clinical Genetics, University Medical Centre Groningen, A. Deusinglaan 4, 9713 AW Groningen, \\ The Netherlands \\ ${ }^{3}$ Laboratory of Molecular Bacteriology, Department of Medical Microbiology, University Medical Centre Groningen and University \\ of Groningen, P.O. Box 30001, 9700 RB Groningen, The Netherlands
}

Bacteria export numerous proteins across the cytoplasmic membrane into the periplasmic space and outer membrane (Gram-negative species) or the extracellular milieu (Gram-positive species). In most bacteria, the bulk of this protein traffic is mediated by the general secretory (Sec) pathway, which requires 'threading' of transported proteins through a membrane-bound Sec translocon in an unfolded state. Upon membrane translocation, exported proteins fold into their final conformation. Recently, a second pathway for protein export has been identified: the twin-arginine translocation (Tat) system, which derives its name from the twin-arginine (RR) motif that is an essential determinant in the signal peptide of Tat-system substrates. This pathway is conserved in Bacteria, Archaea and chloroplast thylakoids. In marked contrast to the Sec system, the Tat system translocates globular proteins in a folded state. This striking characteristic of Tat systems has attracted wide interest and is discussed in excellent reviews by Palmer et al. [1], and Robinson and Bolhuis [2].

In Escherichia coli and chloroplast thylakoids, an active Tat system canonically requires the presence of

Corresponding author: van Dijl, J.M. (j.m.van.dijl@med.umcg.nl).

Available online 21 November 2005 three membrane-bound subunits: TatA, TatB and TatC. Absence of one of these subunits results in impaired function of the Tat machinery, at least with respect to the export of known authentic substrates. The currently accepted view is that, in complex, TatB and TatC serve in RR-signal-peptide reception, whereas, in complex with multiple TatA subunits, they form protein-conducting channels [3,4]. Association of TatA with complexed TatB and TatC subunits requires a functional precursor and the $\Delta \mathrm{pH}$ component of the proton-motive force $[3,4]$. TatA subunits are likely to be recruited by TatB [1]. Here, we highlight the novel finding that TatA has the intrinsic capability to complement for the essential TatB subunit.

Phylogenetic analyses have shown that many bacteria and archaea contain genes that encode multiple TatA-, TatB-, and TatC-like proteins. Known TatA and TatB proteins show limited amino acid sequence similarity (Figure 1). Much clearer similarities between TatA and TatB proteins are, however, observed at the level of their predicted membrane topologies and structures (Figure 2a). A distinctive feature of these proteins is the presence of a conserved motif at the hinge between the transmembrane segment and the amphipathic cytoplasmic helix: Phe-Gly (FG) in TatA and Gly-Pro 